\title{
Predisposição a formas graves de COVID-19 e adesão às medidas de prevenção: o papel do apoio social
}

\author{
Predisposition to severe forms of COVID-19 and adherence \\ to preventive measures: the role of social support
}

Nair Tavares Milhem Ygnatios (https://orcid.org/0000-0002-8862-1930) ${ }^{1}$

Fabíola Bof de Andrade (https://orcid.org/0000-0002-3467-3989) ${ }^{2}$

Maria Fernanda Lima-Costa (https://orcid.org/0000-0002-3474-2980) ${ }^{3}$

Juliana Lustosa Torres (https://orcid.org/0000-0002-3687-897X) ${ }^{1}$
${ }^{1}$ Programa de PósGraduação em Saúde Pública, Universidade Federal de Minas Gerais. Av. Professor Alfredo Balena 190, Santa Efigênia. 30130100. Belo Horizonte MG Brasil.jlt.ufmg@gmail. ${ }^{2}$ Programa de PósGraduação em Saúde Coletiva, Instituto de Pesquisas René Rachou, Fundação Oswaldo Cruz. Belo Horizonte MG Brasil. ${ }^{3}$ Núcleo de Estudos em Saúde Pública e Envelhecimento, Instituto de Pesquisas René Rachou, Fundação Oswaldo Cruz. Belo Horizonte MG Brasil.

\begin{abstract}
This study sought to assess the adherence to preventive measures among the elderly more prone to severe forms of COVID-19, and the association and interaction with social support. It is a cross-sectional study conducted with a sample of 3,477 participants of the telephone survey of the Brazilian Longitudinal Study of Aging (ELSI-COVID-19 initiative), who reported going out of the home in the past week. The adherence was based on the frequency of leaving the house, the need to venture outside the home, use of masks, and sanitization of hands. Statistical analysis was based on the Poisson model with robust variance. Predisposing factors for severe forms of COVID-19 included age $\geq 65$ years, hypertension, diabetes, and obesity. Social support included living arrangements and social distancing during the pandemic. Approximately $46 \%$ of the participants showed higher adherence, which was positively associated with the number of predisposing factors for severe forms of COVID-19. Social support was not associated with adherence, nor was this association modified after adjustments. The conclusion drawn is that higher adherence is concentrated among the elderly with greater predisposition to severe forms of COVID-19, irrespective of social support, albeit preventive measures should be adopted by all.
\end{abstract}

Key words Social support, COVID-19, Predisposing factors, Preventive measures
Resumo Objetivou-se verificar a adesão às medidas de prevenção em idosos com maior predisposição a formas graves de COVID-19 e sua associação e interação com o apoio social. Trata-se de um estudo transversal realizado em amostra de 3.477 participantes do inquérito telefônico do Estudo Longitudinal da Saúde dos Idosos Brasileiros (iniciativa ELSI-COVID-19), que informaram ter saído de casa na semana anterior à realização do inquérito. A adesão foi aferida pela frequência com que saiu de casa, necessidade de sair de casa, uso de máscara e higienização das mãos. As análises basearam-se no modelo Poisson com variância robusta. Idade $\geq 65$ anos, hipertensão, diabetes e obesidade foram considerados fatores predisponentes para formas graves de COVID-19. O apoio social incluiu o arranjo domiciliar e a conexão social na pandemia. Aproximadamente $46 \%$ apresentaram melhor adesão, que foi associada positivamente ao numero de fatores predisponentes para formas graves. O apoio social não foi associado à adesão e não modificou essa associação, após ajustamentos. Conclui-se que a adesão às medidas de prevenção, que deveria ser estendida a todos, está concentrada nos idosos com maior predisposição a formas graves de COVID-19, independentemente do apoio social.

Palavras-chave Apoio social, COVID-19, Fatores predisponentes, Medidas preventivas 


\section{Introdução}

Os coronavírus compreendem uma família de vírus que podem causar resfriados e infecções respiratórias em humanos. Ao considerar os níveis de disseminação e severidade do subtipo 2, ou SARS-CoV-2 (síndrome respiratória aguda grave coronavírus 2), em 11 de março de 2020, a Organização Mundial da Saúde (OMS) declarou pandemia dessa doença, denominada de Coronavírus Disease 2019 (COVID-19), caracterizando-a como uma "emergência de saúde pública de importância internacional"'. Até o dia 09 de janeiro de 2021, o Brasil registrou 8.075.998 casos de COVID-19 e 202.631 óbitos, o que corresponde a uma taxa de letalidade de $2,5 \%{ }^{2}$.

Dados internacionais ${ }^{3-6}$ mostraram que pacientes com obesidade, doenças cardiovasculares, hipertensão arterial, diabetes mellitus, doenças respiratórias crônicas e câncer são mais vulneráveis a desenvolverem formas graves de $\mathrm{CO}$ VID-19. Tanto a obesidade quanto o diabetes mellitus têm demonstrado efeitos negativos no sistema imunológico, devido à exposição crônica a um ambiente metabólico anormal, e que aumenta a suscetibilidade e gravidade de infecções. A obesidade está associada ainda às alterações pulmonares mecânicas e fisiológicas, e promove uma progressão para insuficiência respiratória. Há de se considerar também que a infecção pelo SARS-CoV-2 ocasiona resistência à insulina, piorando o prognóstico do diabetes preexistente ${ }^{7}$.

A partir dessas evidências, países como o Brasil, que apresentam aumento das prevalências dessas Doenças Crônicas Não Transmissíveis (DCNTs) devido ao acelerado processo de envelhecimento populacional, deveriam intensificar ações intersetoriais, e que visaria à redução da demanda e dos riscos de infecção nas unidades de emergência ${ }^{8}$ e a proteção dos idosos que estão sob maior risco de desfechos graves associados a COVID-194: $20 \%$ dos adultos brasileiros apresentam obesidade, enquanto que cerca de $25 \%$ e $7 \%$ relataram diagnóstico médico de hipertensão arterial e diabetes mellitus, respectivamente ${ }^{9}$. Estima-se que $34 \%$ (53 milhões) a $54,5 \%$ (86 milhões) de indivíduos apresentam pelo menos um fator predisponente para formas graves de COVID- $19^{10}$ e que $80 \%$ (34 milhões) dos brasileiros com 50 anos e mais apresentam uma ou mais morbidades ${ }^{11}$.

Nesse contexto, os esforços para reduzir a disseminação de COVID-19 devem ser rigorosos em um país de média-alta renda como o Brasil, que apresenta fragilidades na testagem, bem como limitações na infraestrutura de média e alta complexidade, contribuindo para a sobrecarga do sistema de saúde no enfrentamento à pandemia ${ }^{12}$. Entretanto, a adesão às medidas de prevenção depende não somente das estratégias governamentais, mas também do apoio social recebido pelo indivíduo, fundamental para enfrentar as consequências negativas do distanciamento social $^{13}$. Postula-se que o apoio social adequado ajuda a manter um distanciamento social satisfatório, seja através do apoio emocional, informacional ou instrumental ${ }^{13}$. Assim, objetivou-se verificar: 1) a adesão às medidas de prevenção internacionalmente recomendadas ${ }^{14}$ pelas pessoas com maior predisposição a desenvolverem formas graves de COVID-19;2) se o apoio social está associado à adesão a essas medidas; 3 ) se a adesão a medidas de prevenção varia em função do apoio social.

\section{Métodos}

\section{Fonte de dados e desenho do estudo}

O Estudo Longitudinal da Saúde dos Idosos Brasileiros (ELSI-Brasil) é um estudo de base domiciliar, conduzido em amostra nacional representativa da população brasileira com 50 anos ou mais. A primeira etapa da pesquisa foi conduzida entre 2015 e 2016, com 9.412 participantes, e a segunda etapa entre agosto de 2019 e 17 de março de 2020, com 9.177 participantes. As entrevistas da segunda etapa foram interrompidas na data acima citada, em função da pandemia de COVID-19, por razões éticas, para evitar o potencial de transmissão durante a visita domiciliar.

Em função da pandemia, foi implementada a iniciativa ELSI-COVID-19, baseada em inquérito telefônico com os participantes da segunda etapa, para obter informações sobre os aspectos relacionados à doença. $\mathrm{O}$ inquérito telefônico foi realizado entre 26 de maio e 8 de junho de 2020 , cerca de 60 dias após as estratégias iniciais de distanciamento social no Brasil (com 6.149 participantes $)^{15}$. Maiores detalhes sobre a metodologia do ELSI-Brasil e a metodologia da iniciativa ELSI-COVID-19 podem ser encontrados em publicações anteriores ${ }^{15,16}$.

No presente estudo, foram elegíveis todos os 3.600 participantes do inquérito telefônico que informaram ter saído de casa nos 7 dias anteriores à realização da entrevista, já que somente estes responderam às perguntas referentes às medidas de prevenção relacionadas à COVID-19, 
sendo que 3.477 foram incluídos na análise atual por conterem tais informações completas.

O ELSI-Brasil e a iniciativa ELSI-COVID-19 foram aprovados pelo Comitê de Ética em Pesquisa da Fundação Oswaldo Cruz (Fiocruz), Minas Gerais.

\section{Adesão às medidas de prevenção a COVID-19}

Para avaliar a adesão às medidas de prevenção à COVID-19, adotadas pelos participantes que saíram de casa nos 7 dias anteriores à realização do inquérito telefônico, foram consideradas as seguintes informações: frequência com que saiu de casa ("1 a 2 vezes" ou " $\geq 3$ vezes"); necessidade de sair de casa ("sair por necessidade" é sair para trabalhar, comprar remédios ou alimentos, pagar contas e/ou obter atendimento de saúde, ou "sair sem necessidade"); uso de máscara ao sair de casa ("sempre" ou "às vezes/nunca"); e, higienização das mãos com água e sabão e/ou álcool em gel ao chegar em casa ("sempre” ou "às vezes/nunca”).

Para criação da variável desfecho, a adesão às medidas de prevenção à COVID-19 foi categorizada em "melhor adesão", o que corresponde àqueles que saíram de casa 1 a 2 vezes, por necessidade, sempre usaram máscaras ao saírem de casa e sempre higienizaram as mãos ao chegarem em casa; ou "pior adesão", quando não adotaram essas medidas.

\section{Fatores predisponentes para formas graves de COVID-19}

Foram incluídos quatro fatores predisponentes para formas graves de COVID-19, conforme descritos na literatura ${ }^{10}$ : (1) idade avançada (“<65 anos” ou " $\geq 65$ anos”); diagnóstico médico de (2) hipertensão arterial ("não" ou "sim”) ou (3) diabetes mellitus ("não" ou "sim") autorrelatados, e (4) obesidade. Essas informações foram mensuradas no momento da entrevista presencial dos participantes da segunda etapa do ELSI-Brasil, entre agosto de 2019 e março de 2020.

A obesidade foi classificada de acordo com o Índice de Massa Corporal (IMC) elevado ( $\geq 30$ $\mathrm{kg} / \mathrm{m}^{2}$ ) ("não" ou "sim"), calculado dividindo-se o peso $(\mathrm{kg})$ pela altura $(\mathrm{m})$ ao quadrado ${ }^{17}$. Durante a entrevista presencial, o peso foi aferido por uma balança eletrônica calibrada e a estatura através de um estadiômetro portátil, por meio de procedimentos padronizados, usando roupas leves e sem calçados.
Para as propostas metodológicas, criamos uma variável independente, classificando os participantes de acordo com o número de fatores predisponentes para formas graves de COVID-19 descritos acima (“0”, “1-2" ou "3-4").

\section{Apoio social}

O apoio social incluiu o arranjo domiciliar e a conexão social durante a pandemia. Para o arranjo domiciliar, mensurado na segunda etapa do ELSI-Brasil, foi incluído morar sozinho (a), morar com companheiro (a) e outros arranjos uma vez que idosos que moram sozinho(a) estão menos propensos e os que moram com companheiros(as) mais propensos a receber apoio social $^{18}$. A conexão social durante a pandemia foi baseada nas informações obtidas no inquérito telefônico, considerando-se a frequência de contatos com a família e/ou amigos, que não moram com o indivíduo, por meio da internet, whatsapp ou telefon $\mathrm{e}^{19}$, a considerar os 30 dias anteriores à entrevista telefônica. Ela foi categorizada em "não", se o participante relatou contato social menos de uma vez por semana, ou "sim", se o participante relatou contato social uma ou mais vezes por semana.

\section{Potenciais variáveis de confusão}

Potenciais variáveis de confusão incluíram características sociodemográficas e a limitação funcional, avaliadas na segunda etapa do ELSI -Brasil. Elas incluíram:

Características sociodemográficas, previamente associadas ao distanciamento social e/ou às razões para sair de casa $^{20}$ : sexo ("feminino" ou "masculino"), escolaridade, em anos completos de estudos (" $\leq 8$ anos", "9-11 anos" ou " $\geq$ 12 anos") e regiões brasileiras ("Centro-oeste", "Norte", "Nordeste", "Sudeste" ou "Sul");

Limitação funcional: O desempenho em Atividades Básicas de Vida Diária (ABVDs) foi avaliado por meio do relato de alguma dificuldade para realizar pelo menos uma entre seis ABVDs, incluindo andar de um cômodo para outro, vestir-se, tomar banho, comer, deitar/levantar da cama e usar o banheiro ${ }^{21}$ ("independente", quando não relatou dificuldade em realizar nenhuma das seis atividades ou "dependente").

\section{Análises estatísticas}

Inicialmente foi realizada análise descritiva das variáveis. Foi estimada a frequência da 
adesão às medidas de prevenção relacionadas à COVID-19 para as categorias dos fatores predisponentes para formas graves de COVID-19, do apoio social e das covariáveis e verificada associação por meio do teste do Qui-quadrado de Pearson, com correção de Rao-Scott. A força da associação entre variáveis explicativas de interesse e a adesão às medidas de prevenção à COVID-19 foi avaliada por meio das Razões de Prevalências (RP) brutas e ajustadas, por variáveis de confusão e seus respectivos Intervalos de Confiança de 95\% (IC95\%), estimados pelo modelo de regressão de Poisson com variância robusta. Este modelo foi adotado devido à elevada prevalência de melhor adesão às medidas de prevenção à $\mathrm{CO}-$ VID-19 (46,12\%). Nas análises bruta e ajustada, os ajustes foram feitos da seguinte forma: (1) características sociodemográficas, incluindo sexo, escolaridade e regiões brasileiras (Modelo 1); (2) limitação funcional (Modelo 2); (3) apoio social, que inclui o arranjo domiciliar e a conexão social durante a pandemia (Modelo 3); (4) Modelos 1, 2 e 3 juntos (Modelo 4 - Modelo Ajustado); e, finalmente o (5) Modelo que considera os termos de interação entre cada uma das variáveis de apoio social e número de fatores predisponentes para formas graves de COVID-19, para verificar se o apoio social modifica a associação entre o número de fatores para formas graves e a prevalência de adesão às medidas de prevenção à COVID-19 (Modelo 5). Baseado no modelo 5, o qual considerou os termos de interação, foram plotadas as probabilidades preditas de melhor adesão, de acordo com o número de fatores predisponentes para formas graves de COVID-19 e estratificadas pelo apoio social (arranjo domiciliar e a conexão social durante a pandemia).

As análises foram realizadas com auxílio do software Stata/SE ${ }^{\circledR}$ (Stata Corp., College Station, Estados Unidos), versão 14.0, considerando o delineamento da amostra e os pesos especificamente derivados para os participantes do inquérito telefônico.

\section{Resultados}

Quanto às medidas de prevenção, $46,12 \%$ dos idosos apresentaram melhor adesão. A Tabela 1 mostra a distribuição dos fatores predisponentes, do apoio social, das características sociodemográficas e da limitação funcional de todos os participantes do estudo e de acordo com a adesão às medidas de prevenção à COVID-19. As prevalências de hipertensão arterial e diabetes melli- tus foram de $41,3 \%$ e $15,2 \%$, respectivamente, $30,1 \%$ dos participantes apresentaram obesidade e $29,5 \%$ tinham $\geq 65$ anos. A maioria dos participantes apresentou 1-2 fatores predisponentes para formas graves de COVID-19 (54,9\%), mora com companheiro(a) $(61,8 \%)$ e relatou ter conexão social durante a pandemia $(87,4 \%)$. Além disso, 50,1\% dos participantes tinham menos de 8 anos de escolaridade e $42,2 \%$ eram da região Sudeste. Quanto ao desempenho nas ABVDs, $5,6 \%$ relataram dificuldade em realizar pelo menos uma ABVD. As variáveis: idade $\geq 65$ anos, hipertensão arterial, número de fatores predisponentes para formas graves de COVID-19 e sexo foram estatisticamente diferentes ( $\mathrm{p}$-valor $<0,05$ ) entre as categorias de adesão às medidas de prevenção a COVID-19.

A Tabela 2 mostra os resultados das análises brutas entre cada variável e a adesão às medidas de prevenção, bem como a análise ajustada da associação entre número de fatores predisponentes para formas graves de COVID-19, apoio social e a adesão às medidas de prevenção, ajustadas pelas demais covariáveis. $\mathrm{Na}$ análise ajustada, foi observado que idosos que têm 1-2 fatores predisponentes para formas graves de COVID-19 têm adesão às medidas de prevenção 1,27 vezes a daqueles que não têm fatores predisponentes. Esse valor eleva-se para 1,59 vezes quando os idosos apresentam de 3-4 fatores predisponentes para formas graves de COVID-19. Em relação ao apoio social, a associação observada na análise bruta para quem mora com companheiro (a) $(\mathrm{RP}=0,74$; IC95\% 0,56-0,97) deixou de ser significativa após os ajustamentos. Adicionalmente, observou-se menor propensão de adesão às medidas de prevenção à COVID-19 para os idosos do sexo masculino ( $\mathrm{RP}=0,72$; IC95\% 0,62-0,84), com 9-11 anos de escolaridade ( $\mathrm{RP}=0,85$; IC95\% $0,73-0,98)$ e da região Nordeste $(\mathrm{RP}=0,81 ; \mathrm{IC} 95 \%$ $0,67-0,97)$.

Não foram encontradas interações significativas entre variáveis de apoio social e número de fatores predisponentes para formas graves de COVID-19, mostrando que as associações encontradas para número de fatores predisponentes para formas graves de COVID-19 e adesão às medidas de prevenção não são modificadas pelo apoio social ( $p$-valor $>0,05)$. A Figura 1 mostra as probabilidades preditas de adesão às medidas de prevenção à doença, de acordo com o número de fatores predisponentes para formas graves de COVID-19 e o apoio social (arranjo domiciliar "A" e a conexão social durante a pandemia "B"), baseada no modelo com as interações. Em am- 
bos, observa-se a variação da melhor adesão às medidas de prevenção a COVID-19 em função do número de fatores predisponentes para formas graves de COVID-19 foi semelhante entre as categorias de arranjo domiciliar e conexão social (p-valor $>0,05)$.

\section{Discussão}

Menos da metade dos idosos - aproximadamente $46 \%$ - apresentaram melhor adesão às medidas de prevenção, que foi associada positivamente ao número de fatores predisponentes para formas

Tabela 1. Distribuição das características dos idosos que saíram de casa nos 7 dias anteriores à entrevista, de acordo com a adesão às medidas de prevenção à COVID-19. Estudo Longitudinal da Saúde dos Idosos Brasileiros (iniciativa ELSI-COVID-19), 2020.

\begin{tabular}{|c|c|c|c|}
\hline Variáveis & $\begin{array}{c}\text { Total } \\
\% \\
\end{array}$ & $\begin{array}{c}\text { Pior adesão } \\
\%\end{array}$ & $\begin{array}{c}\text { Melhor adesão } \\
\%\end{array}$ \\
\hline \multicolumn{4}{|c|}{ Fatores predisponentes para formas graves de COVID-19 } \\
\hline Idade $\geq 65$ anos & 29,5 & 23,7 & 36,3 \\
\hline Hipertensão arterial & 41,3 & 35,8 & 47,8 \\
\hline Diabetes mellitus & 15,2 & 12,7 & 18,2 \\
\hline Obesidade & 30,1 & 26,6 & 34,4 \\
\hline \multicolumn{4}{|l|}{ Número de fatores predisponentes } \\
\hline 0 & 32,3 & 37,7 & 25,9 \\
\hline $1-2$ & 54,9 & 53,4 & 56,8 \\
\hline $3-4$ & 12,8 & 8,9 & 17,3 \\
\hline \multicolumn{4}{|l|}{ Apoio social } \\
\hline \multicolumn{4}{|l|}{ Arranjo domiciliar } \\
\hline Mora sozinho (a) & 21,1 & 16,9 & 26,0 \\
\hline Mora com companheiro (a) & 61,8 & 66,7 & 56,2 \\
\hline Outros arranjos & 17,1 & 16,4 & 17,9 \\
\hline \multicolumn{4}{|l|}{ Conexão social durante a pandemia } \\
\hline Não & 12,6 & 13,5 & 11,6 \\
\hline Sim & 87,4 & 86,5 & 88,4 \\
\hline \multicolumn{4}{|l|}{ Características sociodemográficas } \\
\hline \multicolumn{4}{|l|}{ Sexo } \\
\hline Feminino & 50,0 & 41,4 & 59,9 \\
\hline Masculino & 50,0 & 58,6 & 40,1 \\
\hline \multicolumn{4}{|l|}{ Escolaridade } \\
\hline$\leq 8$ anos & 50,1 & 48,1 & 52,4 \\
\hline $9-11$ anos & 11,7 & 13,3 & 9,9 \\
\hline$\geq 12$ anos & 38,1 & 38,6 & 37,7 \\
\hline \multicolumn{4}{|l|}{ Regiões brasileiras } \\
\hline Centro-Oeste & 9,7 & 9,3 & 10,1 \\
\hline Norte & 6,2 & 4,6 & 8,0 \\
\hline Nordeste & 25,1 & 27,4 & 22,4 \\
\hline Sudeste & 42,2 & 42,1 & 42,3 \\
\hline Sul & 16,8 & 16,5 & 17,2 \\
\hline \multicolumn{4}{|l|}{ Limitação funcional } \\
\hline \multicolumn{4}{|l|}{ Desempenho nas ABVDs } \\
\hline Independente & 94,4 & 95,7 & 92,9 \\
\hline Dependente & 5,6 & 4,3 & 7,1 \\
\hline Total $(\mathrm{n})^{1}$ & 3.477 & 1.760 & 1.717 \\
\hline
\end{tabular}

${ }^{1}$ Número de entrevistados, sem incluir as correções de acordo com os parâmetros de amostragem.

Em negrito: $p$-valor $<0,05$, com base no teste do Qui-quadrado de Pearson, com correção de Rao-Scott.

Fonte: Elaborado pelos autores. 
graves de COVID-19 e não foi associada ao apoio social, após ajustamentos. Além disso, o apoio social não modificou a associação entre melhor adesão e número de fatores predisponentes para formas graves de COVID-19.

Sem vacinas disponíveis até o momento e nenhum tratamento eficaz, aliado ao escasso conhecimento sobre os modos de disseminação do SARS-CoV-19 por portadores assintomáticos, reduzir a taxa de transmissão e infecção pelo SARS-CoV-2, através da adoção de medidas preventivas, é uma prioridade em Saúde Pública. Evidências incluídas em uma revisão sistemática e metanálise apoiam que o distanciamento social, o uso de máscara e a higienização das mãos são necessários para evitar a infecção na comunidade $^{22}$. Publicações anteriores apresentaram os resultados da primeira série dos inquéritos telefônicos da iniciativa ELSI-COVID-19, estimando as prevalências das medidas de prevenção a COVID-19 e suas associações com características sociodemográficas ${ }^{20}$ e multimorbidades ${ }^{23}$. A pre-

Tabela 2. Associação bruta e ajustada entre os fatores associados à adesão às medidas de prevenção à COVID-19, em idosos que saíram de casa nos 7 dias anteriores à entrevista. Estudo Longitudinal da Saúde dos Idosos Brasileiros (iniciativa ELSI-COVID-19), 2020.

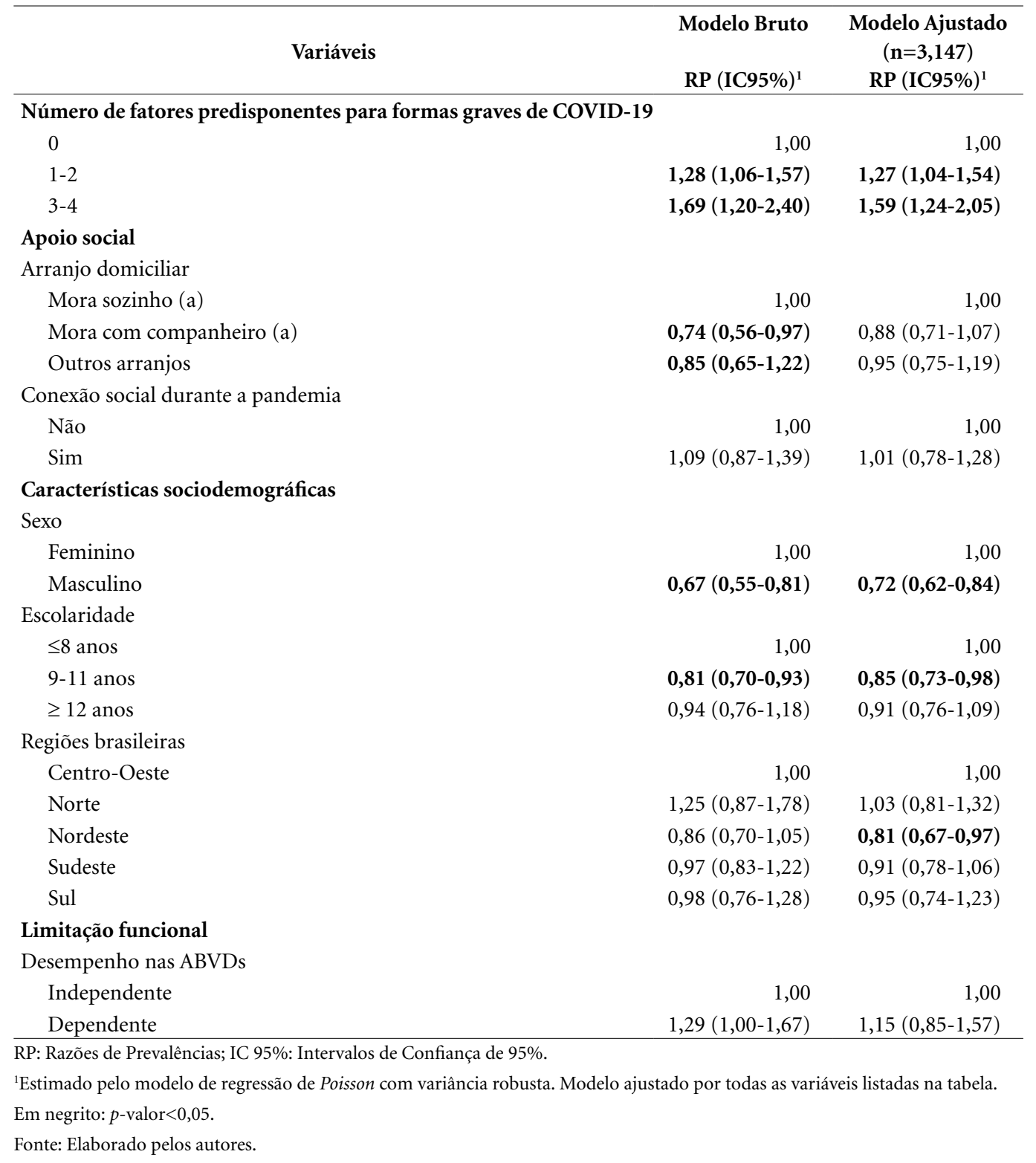


sente análise acrescenta as demais informações quanto a não influência do apoio social na adesão às medidas de prevenção entre idosos, que é maior entre aqueles com mais fatores predisponentes para formas graves de COVID-19.

Um dos achados mais importantes da presente análise foi a forte associação entre número de fatores predisponentes para formas graves de COVID-19 e adesão às medidas de prevenção, em que a prevalência de melhor adesão aumenta à medida que aumenta o número de fatores predisponentes para formas graves de COVID-19. A doença do coronavírus apresenta-se como um risco para a população mundial e as pessoas podem estar cientes do risco real, considerando a repercussão das informações nos meios de comunicação. Alguns estudos documentaram associação entre percepção de risco e adoção de medidas preventivas na epidemia da Síndrome Respiratória Aguda Grave (SARS), em 2003 24,25. Essa hipótese pode ser levantada para explicar os resultados encontrados na presente análise.

Apesar da grande necessidade de introdução dessas medidas de prevenção, em especial na presença de fatores predisponentes para formas graves de COVID-1926, elas podem gerar um impacto negativo na saúde. Um estudo realizado com idosos americanos comprovou que a solidão aumentou durante o distanciamento social ${ }^{27}$. Os indivíduos podem sofrer de estresse e ansiedade, decorrentes da limitação das interações sociais e medo das consequências da infecção à saúde ${ }^{28}$. A longo prazo, o isolamento domiciliar pode estar associado à limitação na continuação da prestação de cuidados às DCNTs preexistentes, apoio e tratamento personalizados ${ }^{29}$.

Pelo exposto acima, hipotetizou-se que a presença de apoio social, mensurado pelo arranjo domiciliar e a conexão social durante a pandemia, modificaria a adesão às medidas preventivas, pois ampliaria o apoio emocional, informacional e instrumental disponíveis para manter o distanciamento social e prevenir suas consequências negativas ${ }^{27,29,30}$. Com o distanciamento social, os idosos podem ter diminuído o contato com a família e amigos que não moram no domicílio e, consequentemente, podem ter piorado sua saúde mental ${ }^{27,31}$. Entretanto, o tipo de arranjo familiar e a conexão social não modificaram a adesão às medidas de prevenção, o que mostra que os indi-
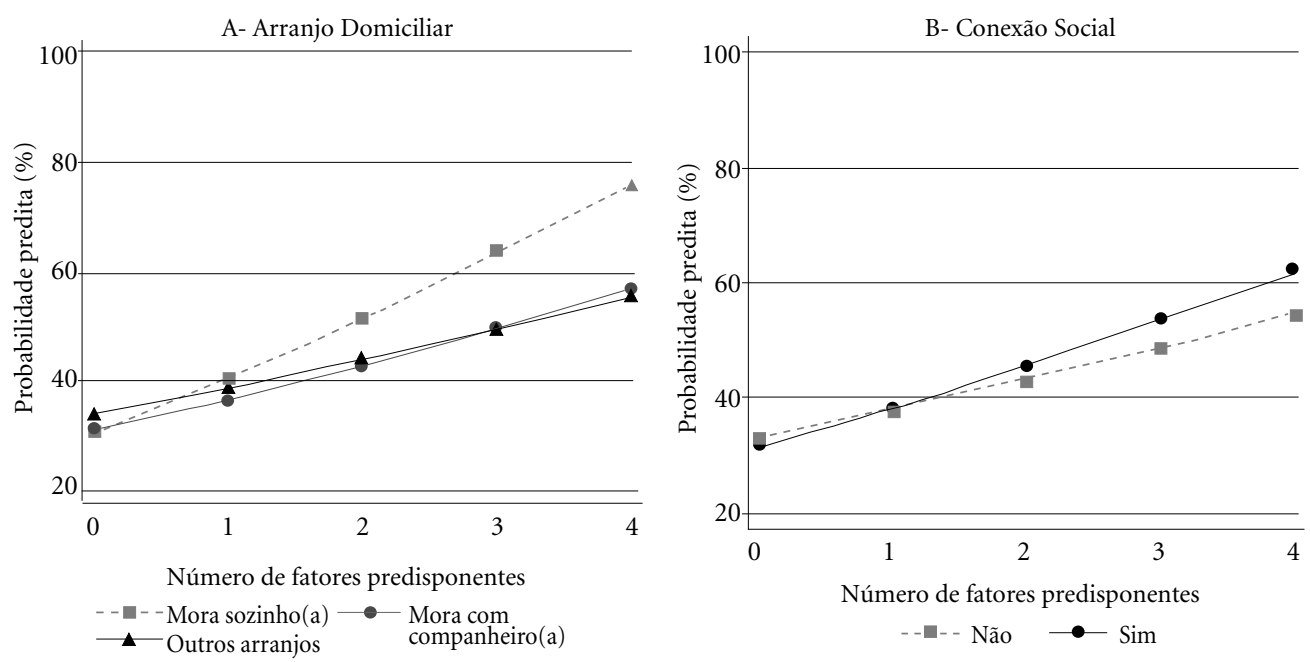

Figura 1. Probabilidade predita* de adesão às medidas de prevenção a COVID-19, de acordo com o número de fatores predisponentes para formas graves de COVID-19 e o apoio social (arranjo domiciliar (A) e conexão social durante a pandemia (B)), em idosos que saíram de casa na última semana. Estudo Longitudinal da Saúde dos Idosos Brasileiros (iniciativa ELSI-COVID-19), 2020.

\footnotetext{
${ }^{\star}$ Estimada pelo modelo de regressão de Poisson com variância robusta, ajustado pelas características sociodemográficas, incluindo sexo, escolaridade e regiões brasileiras; limitação funcional; e, os termos de interação entre apoio social, incluindo arranjo domiciliar e a conexão social durante a pandemia e fatores predisponentes para formas graves de COVID-19.
} 
víduos têm recebido o apoio social equitativo, independentemente do número de fatores predisponentes para formas graves de COVID-19. Esses resultados vão ao encontro das recomendações da OMS no controle da pandemia, publicadas em 14 de abril de 2020 no documento COVID19 Strategy Uptadate ${ }^{14}$, no qual a equidade deve ser um dos princípios norteadores das medidas porque todos estão em risco até que o vírus seja controlado mundialmente.

No entanto, independentemente de não influenciar a adesão às medidas de prevenção à COVID-19, ações que fortaleçam o apoio social durante a pandemia, como a utilização dos meios digitais diversos (telefone, e-mail, mídia social ou videoconferência) ${ }^{32}$ e a continuidade do cuidado das DCNT pela Atenção Primária à Saúde (APS), devem ser estimuladas para minimizar os impactos negativos da pandemia na saúde e, inclusive, diminuir a demanda nas redes de atenção à saú$\mathrm{de}^{33}$. No Brasil, os estudos sobre o apoio social no bem-estar e saúde durante a pandemia ainda são limitados, mas, achados internacionais sugerem essa relação: idosos que moram sozinhos relataram maiores sentimentos negativos, menor qualidade de sono durante a COVID- $19^{30}$, apesar de revelarem aumento da conexão social virtual ${ }^{27}$.

$A$ adesão às medidas de prevenção depende da influência de aspectos socioeconômicos, culturais e, principalmente, do rigor das medidas políticas obrigatórias ${ }^{34,35}$. Deste modo, os governos devem adotar medidas obrigatórias para melhorar a adesão do que esperar que os indivíduos o façam por conta própria ${ }^{35}$, principalmente porque nossos achados mostram que a adesão, no Brasil, está mais concentrada naqueles em maior predisposição.

Por fim, algumas limitações potenciais são observadas neste estudo. Em primeiro lugar, deve-se ressaltar que a pesquisa foi realizada por entrevista telefônica, o que diminui taxa de respostas $(67 \%)$ devido à inexistência de números válidos, não atendimento às chamadas ou recusa em prestar informações pelo telefone, o que é inerente a todos os inquéritos realizados por este meio. Para contornar isso, foram construídos pesos especificamente derivados para os respondentes ao inquérito telefônico ${ }^{15}$. Em segundo lugar, os arranjos domiciliares referem-se aos dos meses anteriores à pandemia. Eventuais mudanças podem ter ocorrido, mas infelizmente os arranjos domiciliares no momento da pandemia não estão disponíveis no inquérito ELSICOVID-19. Em terceiro lugar, embora tenhamos feito controle para muitas covariáveis, não podemos excluir a possibilidade de algum confundimento residual causado por fatores não medidos. Finalmente, este é um estudo transversal, por isso, não é possível elucidar as relações causais.

Entre os pontos fortes, destaca-se o monitoramento de uma amostra nacionalmente representativa de idosos brasileiros que contribui para a generalização dos resultados para essa população e, na validade interna do estudo, dado o rigor dos procedimentos metodológicos ado$\operatorname{tados}^{15}$. Os próximos inquéritos telefônicos previstos pela iniciativa ELSI-COVID-19 permitirão acompanhar possíveis mudanças na adesão às medidas de prevenção à doença, em função da flexibilização das medidas no país.

\section{Conclusão}

Em conclusão, é necessário um esforço comum para a maior adesão às medidas de prevenção a COVID-19. Os presentes resultados permitem comprovar que a adesão às medidas de prevenção variam entre os subgrupos e está concentrada nos idosos em maior risco de formas graves de COVID-19, enquanto que tais medidas deveriam ser feitas por todos. Cabe aos profissionais da saúde e ao governo orientar a população quanto à importância da manutenção das medidas de prevenção, independentemente da presença de fatores predisponentes. Apesar de a maioria dos idosos relatarem conexão social durante a pandemia, devem-se implantar estratégias que minimizem possíveis impactos negativos do distanciamento social na sua saúde mental. 


\section{Colaboradores}

MF Lima-Costa, JL Torres e FB Andrade conceberam o estudo. MF Lima-Costa, JL Torres, FB Andrade e NTM Ygnatios contribuíram para o desenho do estudo, a análise e a interpretação dos dados e a redação do artigo. Todos os autores revisaram e aprovaram a versão final.

\section{Financiamento}

O ELSI-Brasil foi financiado pelo Ministério da Saúde: Departamento de Ciência e Tecnologia da Secretaria de Ciência, Tecnologia e Insumos Estratégicos (DECIT/SCTIE) e pela Coordenação da Saúde da Pessoa Idosa, Departamento de Estratégia e Ações Programáticas da Secretaria de Atenção à Saúde (COSAPI/DAPES/SAS). A iniciativa ELSI-COVID-19 foi financiada pelo DECIT/SCTI e pelo Conselho Nacional de Desenvolvimento Científico e Tecnológico (CNPq).

\section{Agradecimentos}

Os autores agradecem aos participantes e aos profissionais que colaboraram para a realização do ELSI-Brasil e iniciativa ELSI-COVID-19.

\section{Referências}

1. World Health Organization (WHO). Timeline of WHO's response to COVID-19 [internet]. Geneva: WHO; 2020. [cited $2021 \mathrm{Feb}$ 19]. Available from: https://www.who.int/news-room/detail/29-06-2020covidtimeline.

2. Ministério da saúde (MS). COVID-19 Painel Coronavírus. Brasília: MS; 2021 [acessado 2020 nov 9]. Disponível em: https://covid.saude.gov.br/

3. Wu Z, McGoogan JM. Characteristics of and important lessons from the coronavirus disease 2019 (COVID-19) outbreak in China: summary of a report of 72314 cases from the chinese center for disease control and prevention. J Am Med Assoc 2020; 323(13):1239-1242.

4. Chow N, Fleming-Dutra K, Gierke R, Hall A, Hughes M, Pilishvili T, Ritchey M, Roguski K, Skoff T, Ussery E. Preliminary estimates of the prevalence of selected underlying health conditions among patients with coronavirus disease 2019 - United States, February 12-March 28, 2020. Morb Mortal Wkly Rep 2020; 69(13):382-386.

5. Yang J, Hu J, Zhu C. Obesity aggravates COVID-19: a systematic review and meta-analysis. J Med Virol 2020; 93(1):257-261.

6. Li B, Yang J, Zhao F, Zhi L, Wang X, Liu L, Bi Z, Zhao Y. Prevalence and impact of cardiovascular metabolic diseases on COVID-19 in China. Clin Res Cardiol 2020; 109(5):531-538.

7. Zhou Y, Chi J, Lv W, Wang Y. Obesity and diabetes as high-risk factors for severe coronavirus disease 2019 (COVID-19). Diabetes Metab Res Rev 2020; 37(2):e3377.

8. Daumas RP, Silva GA e, Tasca R, et al. O papel da atenção primária na rede de atenção à saúde no Brasil: limites e possibilidades no enfrentamento da COVID-19. Cad Saude Publica 2020; 36(6):e00104120.

9. Ministério da Saúde (MS). Secretaria de Vigilância em Saúde. Vigitel Brasil 2019: vigilância de fatores de risco e proteção para doenças crônicas por inquérito telefônico: estimativas sobre frequência e distribuição sociodemográfica de fatores de risco e proteção para doenças crônicas nas capitais dos 26 estados brasileiros e no Distrito Federal em 2019 [internet] / Ministério da Saúde, Secretaria de Vigilância em Saúde, Departamento de Análise em Saúde e Vigilância de Doenças não Transmissíveis. Brasília: MS, 2020. 137 p.

10. Rezende LFM, Thome B, Schveitzer MC, Souza-Júnior PRB, Szwarcwald CL. Adults at high-risk of severe coronavirus disease-2019 (Covid-19) in Brazil. Rev Saude Publica [serial on the internet] 2020 [cited 2020 Nov 5]; 54(50). Available from: http:// www.scielo.br/scielo.php?script=sci_arttext\&pi$\mathrm{d}=$ S0034-89102020000100239\&lng=en\&nrm=iso

11. Nunes BP, Souza ASS, Nogueira J, Andrade FB, Thumé E, Teixeira DSC, Lima-costa MF, Facchini LA, Batista SR. Envelhecimento, multimorbidade e risco para COVID-19 grave : ELSI-Brasil [preprint]. 2020 [cerca de 14 p.] [Acessado $2021 \mathrm{fev}$ 15]. Disponível em: https://preprints.scielo.org/index.php/scielo/pre print/view/703/929

12. Oliveira WK, Duarte E, França GVA, Gracia LP. Como o Brasil pode deter a COVID-19. Epidemiol e Serv saude 2020; 29(2):e2020044. 
13. Hwang TJ, Rabheru K, Peisah C, Reichman W, Ikeda M. Loneliness and Social Isolation during the COVID-19 Pandemic. Int Psychogeriatrics 2020; 32(10):1217-1220.

14. World Health Organization (WHO). COVID-19 Strategy Up Date. Geneva: WHO; Apr. 2020 [cited 2021 Fev 20]. Available from: https://www.who.int/docs/ default-source/coronaviruse/covid-strategy-update -14april2020.pdf?sfvrsn=29da3ba0_19\&download $=-$ true

15. Lima-Costa MF, Macinko J, Andrade FB, Souza júnior PRB, Vasconcellos MTR, Oliveira CM. ELSICOVID-19 initiative: methodology of the telephone survey on coronavirus in the Brazilian Longitudinal Study of Aging. Cad. Saude Publica 2020; 36(Supl. 3):e00183120.

16. Lima-Costa MF, Andrade FB, Souza-Júnior PRB, Neri AL, Duarte YAO, Castro-Costa E, Oliveira C. The brazilian longitudinal study of aging (ELSI-Brazil): objectives and design. Am J Epidemiol 2018; 187(7):1345-1353.

17. World Health Organization (WHO). Obesity: Preventing and managing the global epidemic. Geneva: WHO; 2020. [cited $2021 \mathrm{Feb} 20$ ]. Available from: https://www.who.int/nutrition/publications/obesity/ WHO_TRS_894/en/ (2000).

18. Duarte YAO, Lebrão ML, Lima FD. The contribution of living arrangements in the provision of care for elderly persons with functional impairments in São Paulo, Brazil. Rev Panam Salud Publica/Pan Am J Public Heal 2005; 17(5-6):370-378.

19. Berkman LF, Glass T, Brissette I, Seeman TE. From social integration to health: Durkheim in the new millennium. Soc Sci Med 2000; 51(6):843-857.

20. Lima-Costa MF, Mambrini JVM, Andrade FB, Peixoto SWV, Macinko J. Distanciamento social, uso de máscaras e higienização das mãos entre participantes do estudo longitudinal da saúde dos idosos brasileiros: iniciativa ELSI-COVID-19. Cad Saude Publica 2020; 36(Supl. 3):1-13.

21. Katz S, Akpom CA. A measure of primary sociobiological functions. Int J Heal Serv 1976; 6(3):493-508.

22. Chu DK, Akl EA, Duda S, Solo K, Yaacoub S, Schünemann HJ on behalf of the C-19 SUR, Authors GE (SURGE) study. Physical distancing, face masks, and eye protection to prevent person-to-person transmission of SARS-CoV-2 and COVID-19: a systematic review and meta-analysis. Lancet 2020; 395(issue 10242):1973-1987.

23. Batista SR, Souza ASS, Nogueira J, Andrade FB, Thumé E, Teixeira DSC, Lima-Costa MF, Facchini LA, Nunes BP. Comportamentos de proteção para COVID-19 entre adultos e idosos brasileiros que vivem com multimorbidade (Iniciativa ELSI-COVID-19) Cad Saude Publica 2020; 36(15):1-14 [Acessado 2021 fev 15]. Disponível em: https://preprints.scielo.org/ index.php/scielo/preprint/view/1027/1470

24. Brug J, Aro AR, Richardus JH. Risk perceptions and behaviour: Towards pandemic control of emerging infectious diseases: Iional research on risk perception in the control of emerging infectious diseases. Int $J$ Behav Med 2009; 16(1):3-6.

25. Blendon RJ, Benson JM, DesRoches CM, Raleigh E, Taylor-Clark K. The public's response to severe acute respiratory syndrome in Toronto and the United States. Clin Infect Dis 2004; 38(7):925-931.
26. Pititto BA, Ferreira SRG. Diabetes and covid-19: more than the sum of two morbidities. Rev Saude Publica [serial on the internet] 2020 [cited 2020 Nov 5]; 54(54). Available from: http://www.scielo.br/scielo. php?script $=$ sci_arttext $\&$ pid $=$ S0034 -89102020000100 603\&lng=en\&nrm=iso

27. Emerson KG. Coping with being cooped up: Social distancing during COVID-19 among 60+ in the United States. Rev Panam Salud Publica/Pan Am J Public Heal [serial on the internet] 2020 [cited 2020 Nov 5]; 44(e81). Available from: https://doi.org/10.26633/RP SP.2020.81

28. Xiao H, Zhang Y, Kong D, Li S, Yang N. Social capital and sleep quality in individuals who self-isolated for 14 days during the coronavirus disease 2019 (COVID-19) outbreak in January 2020 in China. Med Sci Monit 2020; 26:e923921-1 - e923921-8.

29. Szcze D, Anna G. The SARS-CoV-2 and mental health: From biological mechanisms to social consequences. Prog Neuropsychopharmacol Biol Psychiatry 2021;104:110046.

30. Goodman-Casanova JM, Dura-Perez E, Guzman-Parra J, Cuesta-Vargas A, Mayoral-Cleries F. Telehealth home support during COVID-19 confinement for community-dwelling older adults with mild cognitive impairment or mild dementia: survey study. J Med Internet Res 2020; 22(5):e19434.

31. Kuiper JS, Zuidersma M, Voshaar RCO, Zuidema SU, Heuvel ERVD, Stolk RP, Smidt N. Social relationships and risk of dementia: a systematic review and meta-analysis of longitudinal cohort studies. Ageing Res Rev 2015; 22:39-57.

32. World Health Organization (WHO). Mental health and psychosocial considerations during the COVID-19 outbreak. Geneva: WHO; 2020 [cited 2021 Fev 20]. Available from: https://apps.who.int/iris/ bitstream/handle/10665/331490/WHO-2019-nCoV-MentalHealth-2020.1-eng.pdf? sequence $=1 \& \mathrm{i}-$ sAllowed $=\mathrm{y}$

33. Guedes MBOG, Lima KC, Caldas CP, Veras RP. Apoio social e o cuidado integral à saúde do idoso. Physis 2017; 27(4):1185-1204.

34. Aquino R, Souza-Filho JA, Rocha AS, Ferreira A, Victor A, Teixeira C, Machado DB, Paixão E, Alves FJO, Pilecco F, Menezes G, Gabrielli L, Leite L, Almeida MCC, Ortelan N, Fernandes QHRF, Ortiz RJF, Palmeira RN, Pinto Junior EP, Aragão E, Souza LEPF, Barral Netto M, Teixeira MG, Barreto ML, Ichihara MY, Lima RTRS. Social distancing measures to control the COVID-19 pandemic: Potential impacts and challenges in Brazil. Cien Saude Colet 2020; 25(Supl. 1):2423-2446.

35. Moraes RF. Determinants of physical distancing during the covid-19 epidemic in Brazil: effects from mandatory rules, numbers of cases and duration of rules. Cien Saude Colet 2020; 25(9):3393-3400.

Artigo apresentado em 10/11/2020

Aprovado em 01/02/2021

Versão final apresentada em 03/02/2021

Editores-chefes: Romeu Gomes, Antônio Augusto Moura da Silva 